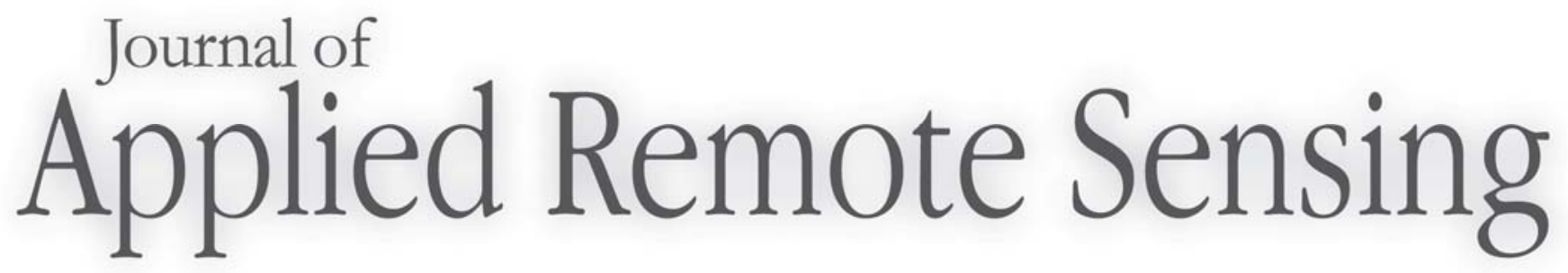

\title{
Lidar detected spike returns
}

\author{
William P. Hooper \\ Glendon M. Frick
}

\section{O SPIE}




\title{
Lidar detected spike returns
}

\author{
William P. Hooper and Glendon M. Frick \\ Naval Research Laboratory, Remote Sensing Division, Washington DC 20735, USA \\ bill.hooper@nrl.navy.mil
}

\begin{abstract}
Lidar measurements of spike returns from clear air are presented. These spikes occur infrequently (approximately one in hundred returns) but provide returns that are significantly stronger (occasionally an order of magnitude larger) than the average aerosol backscatter signal. The spike density is $5.7 \mathrm{e}-3$ spikes $\mathrm{m}^{-3}$ for backscattering cross sections estimated to be between 0.003 and $0.080 \mathrm{~mm}^{2} \mathrm{sr}^{-1}$. A modified form of the lidar equation which includes returns from large particulates is presented and the probability distribution for the spike magnitudes is derived from five million measurements.
\end{abstract}

Keywords: lidar, near-infrared, insect, large aerosols, ultra-large aerosols, spike returns.

\section{INTRODUCTION}

Strong isolated returns have been noted from the earliest days of active remote sensing. In the 1940's when radars were being developed, strong "spike" returns from clear air, where no return was expected, were whimsically called "angels" [1]. Measurements showed that the characteristics of the angels depended on the radar transmit wavelength and were caused by hard target returns or "spot angels" from birds [2], insects [3], and more diffuse returns from refractive structures [1].

Early lidars also occasionally detected spikes from birds, insects, hydrometeors and ice particles; however, since much of the research focused on volumetric scattering from large numbers of smaller aerosols which impact atmospheric optics, these strong returns were normally not reported. Unlike radar, lidar does not detect scattering from refractive structures [4] and thus the lidar returns are caused by either molecules or aerosol particles. (In this paper, the term "aerosols" will denote small atmospheric particles and the term "particulates" will denote larger particles, insects and birds).

The spike returns discussed in this paper are strong isolated returns detected by a shortpulse, incoherent lidar. Figure 1 shows examples of the largest and smallest spikes. These isolated spike returns were detected in clear air while testing an eye-safe lidar. The spike returns are significantly stronger than background scattering and occurred with no visually apparent scattering source. Initially concerns were that the spike returns could be caused by instrumentation and the detector, detector power supplies, and the digitzer were tested and yielded no instrument problems that could generate spike returns. Since the spikes occurred at the same ranges that returns are seen from atmospheric aerosol structures, the larger spikes are apparently caused by scattering from one or more "sub-visible" particulate targets and contrast with the smaller backscatter signals from nearby measurement volumes that only have the smaller aerosols and have no large particulates.

In this paper, we will discuss the strength of these returns and their probability of occurrence. Section 2 discusses the lidar including features which may explain the spike detection. Section 3 discusses a modified lidar equation and the data analysis technique used to characterize the spike returns. Section 4 discusses the measurements and finally section 5 summarizes the measurements results. 


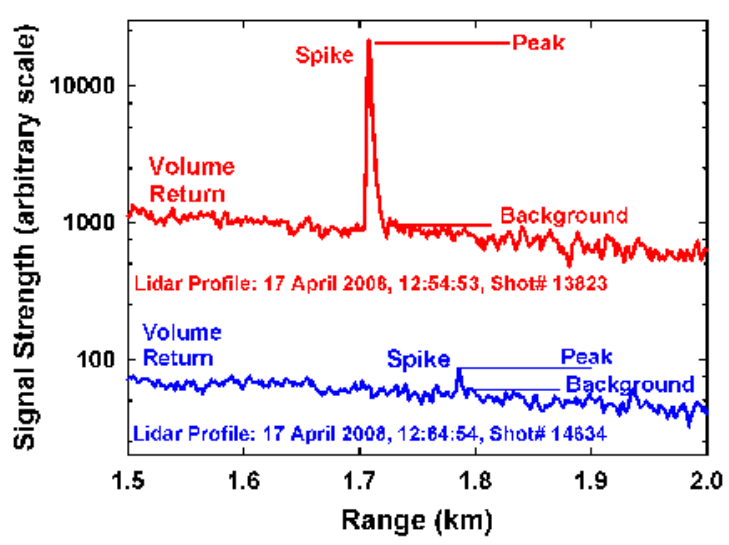

Fig. 1. Lidar profiles showing the largest and smallest spike returns. The background signal in the blue curve is similar to the background signal in the red curve; however, the blue curve was scaled to offset it from the red curve and allow both curves to be plot together.

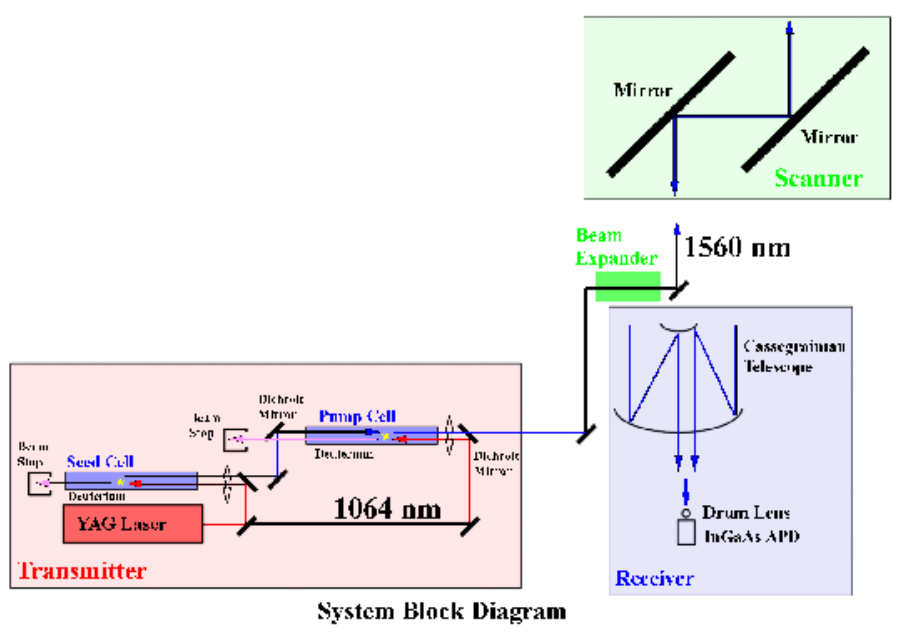

Fig. 2. Schematic of the NRL Eye-safe Volume Imaging Lidar (NEVIL) System.

\section{INSTRUMENT DESCRIPTION}

An existing scanning lidar, which has been used in a series of field experiments (for example, see Hooper and Martin, 1999) [5], has been modified to form an eye-safe lidar. The original, non-eye-safe transmitter used a Nd:YAG laser to transmit at $1064 \mathrm{~nm}$. The new eye-safe system is build around coupled deuterium cells [6] that shift the optical pulses at $1064 \mathrm{~nm}$ to $1560 \mathrm{~nm}$. Figure 2 shows the schematic and Table 1 lists the parameters for the NRL Eye-safe Volume Imaging Lidar (NEVIL) system. The Nd:YAG laser pulse is split between two cells: thirty percent is directed into the seed cell with the remaining energy directed into the pump cell. A backward-propagating beam is generated by the seed cell, amplified and combined with the pulse from the pump cell which provides a light pulse that should replicate the divergence of the original Nd:YAG laser beam. The Nd:YAG laser is injection seeded, which 
reduces the laser line width; a narrow line width is essential and allows the Raman conversion processes to have an operating efficiency of $20 \%$ or better. When we measure the beam quality, we find a beam divergence of $100 \mu \mathrm{rad}$ or less, which differs from the value that we expect. It is possible that the Raman conversion process is more efficient for the more highly collimated photons and thus changes the divergence of the output beam. Since the pulses counter propagate, the output pulse is shorter ( $3 \mathrm{~ns}$ ) than the original Nd:YAG pulse (8 ns). A Galilean beam expander increases the beam diameter from approximately $2 \mathrm{~cm}$ to $6 \mathrm{~cm}$. The resulting $1560 \mathrm{~nm}$ beam is transmitted into the atmosphere.

Table 1. NRL Eye-safe Volume Imaging Lidar (NEVIL) System Parameters

\begin{tabular}{|l|l|}
\hline Transmitter & Raman-shifted Nd:YAG \\
\hline Type & Continuum 8010 (injection seeded) \\
\hline Pump laser & $0.003 \mathrm{~cm}^{-1}$ \\
\hline Pump linewidth & $1560 \mathrm{~nm}$ \\
\hline Output Wavelength & $3 \mathrm{~ns}$ \\
\hline Pulse length & $200 \mathrm{~mJ} /$ pulse \\
\hline Energy & $10 \mathrm{~Hz}$ \\
\hline Repetition Rate & $100 \mu$ rad (full width at half maximum) \\
\hline Divergence (for output beam) & \\
\hline Receiver & Cassegrainian (diameter 0.36 m, focal ratio f/11) \\
\hline Telescope & InGaAs APD, diameter $200 \mu \mathrm{m}$ \\
\hline Detector & $\begin{array}{l}\text { Drum Lens (BK-7, } 4 \text { mm diameter, curvature leading } \\
\text { surface } 2.5 \text { mm) }\end{array}$ \\
\hline Detector Collecting Optics & $100 \mu$ rad \\
\hline Field of View & $50 \mathrm{MHz}, 12$ bit \\
\hline Digitizer &
\end{tabular}

The light backscattered from the atmosphere reflects off a dual mirror scanning system, is collected by a $0.3 \mathrm{~m}$ Cassegrainian telescope and the backscattered signal is focused onto a $200 \mu \mathrm{m}$ diameter InGaAs avalanche photodiode (APD). A small drum lens is mounted directly above the APD and used to increase the APD collection efficiency. (Drum lens are machined from ball lens into a cylinders and are easier to align with cylindrical detector packages.) The receiver field of view is approximately $100 \mu \mathrm{rad}$.

The lidar system was tested on a $400 \mathrm{~m}$ range. During these tests, we found that the beam divergence was also approximately $100 \mu \mathrm{rad}$ and that the energy distribution across the beam has a Gaussian distribution. The transmit beam fully illuminates the receiver field of view; thus, while the backscatter signal from particulates from the center of measurement volume is stronger than the signal from the side edges of the measurement volume, both signals should be detectable if the particulate backscatter is significantly stronger than the background aerosol signal. Therefore, we assume that the measurement volume $\left(\pi r^{2}(\Delta \varphi)^{2} \Delta r\right)$ is determined by the range (r), the receive the field of view, where the angle $(\Delta \varphi \sim 50 \mu \mathrm{rad})$ is half the receiver field of view, and the range resolution $(\Delta \mathrm{r})$.

Prior to our measurement runs, a green laser pointer is placed in the far field and is aimed at 
the lidar. The green laser is focused both onto the InGaAS APD detector and into the D2 cells of the transmitter. With the pump cell removed, a pinhole is placed at the location of the focus spot for the $1064 \mathrm{~nm}$ YAG beam. When the focused green beam is focused both on the detector and the center of the Raman cell, the system is aligned. This alignment maximizes the overlap function of the transmitter and receiver.

The new, eye-safe lidar differs from the old non-eye-safe system in that: the wavelength is shifted from $1064 \mathrm{~nm}$ to $1560 \mathrm{~nm}$, the pulse length is reduced from $8 \mathrm{~ns}$ to $3 \mathrm{~ns}$, beam divergence is reduced from 1 milliradian to $100 \mu \mathrm{rad}$, and range resolution is reduced from $7.5 \mathrm{~m}$ to $1.5 \mathrm{~m}$. These changes allow the detection of small aerosol structures, such as those generated by breaking waves. However as we explain in the following two sections, these changes also make the spike returns from large particulates significantly more detectable.

\section{THEORY}

Lidars operating in the near-infrared receive backscatter signals from molecules and aerosols. All the measurements presented in this paper made within $300 \mathrm{~m}$ of the surface and are within the Earth's boundary layer where the aerosol signal is especially strong. The lidar equation for volumetric aerosol scattering is [7]:

$$
P(r)=K r^{-2} \beta_{T}(r) \Delta r \exp \left(-2 \int_{r^{\prime}=0}^{r} \varepsilon\left(r^{\prime}\right) d r^{\prime}\right)+n(r),
$$

where $\mathrm{P}$ is power return for volume backscatter, $\mathrm{K}$ contains system constants and laser output energy, $\beta_{\mathrm{T}}$ is total volume backscatter coefficient from all aerosols and particulates, $\varepsilon$ is the volume extinction coefficient, and $\mathrm{n}$ is the offset caused by sky and electronic noise. The volume backscatter and extinction coefficient are impacted by both aerosol and molecules; however in the atmospheric boundary layer, the molecular backscatter and extinction in the near-infrared are typically more than a hundred times weaker than the aerosol backscatter and extinction; thus, the molecular scattering can be neglected.

The density of aerosols depends strongly on the size of the particles. In the atmosphere's clear air boundary layer, there are often billions of small particles (less than fifty microns in size) in a cubic meter and a very small number of particles larger than fifty microns in a cubic meter (in fact this density may be substantially less than one). The volume backscatter can be arbitrarily split between the volumetric scattering from large number of small particles and the sum of scatters from a small number the large particulates:

$$
\beta_{T}(r)=\beta_{o}(r)+\pi^{-1} r^{-2}(\Delta \varphi)^{-2}(\Delta r)^{-1} \sum_{i=1}^{N} \sigma_{\pi i},
$$

where $\beta_{0}$ is the volume backscatter coefficient from smaller particles (aerosols), $i$ is the index for the particulates, $\mathrm{N}$ is the total number of particulates in the measurement volume, and $\sigma_{\pi i}$ is the backscattering cross section for $\mathrm{i}^{\text {th }}$ particulate (with units of $\mathrm{mm}^{2} \mathrm{sr}^{-1}$ ). For this calculation, the measurement volume is assumed to be uniformly illuminated, see the discussion in Section 2. The backscattering cross section is defined as the differential scattering cross section [8]:

$$
\sigma_{\pi}=\frac{d \sigma_{T}(\pi)}{d \Omega}
$$

where $\sigma_{\mathrm{T}}$ is total scattering and the derivative is taken for backscattering into the solid angle 
element $\mathrm{d} \Omega$.

Using Eq. (2), an alternative form of the lidar equation is:

$$
P(r)=K r^{-2}\left(\beta_{o} \Delta r+\pi^{-1} r^{-2}(\Delta \varphi)^{-2} \sum_{i=1}^{N} \sigma_{\pi i}\right) \exp \left(-2 \int_{r^{\prime}=0}^{r} \varepsilon\left(r^{\prime}\right) d r^{\prime}\right)+n(r) .
$$

Equation (4) explains, in part, why the new eye-safe lidar detects spike returns when the older system did not. As the range resolution and the divergence angle decrease, the volume backscatter term $\left(\beta_{0} \Delta r\right)$ decreases and the particulate scattering term $\left(\pi^{-1} r^{-2}(\Delta \varphi)^{-2}\right)$ increases and the ratio of the particulate scattering term to backscatter term is about five hundred times larger for the new eye-safe lidar than for the older lidar.

If there is only a single large particulate in a measurement volume, the backscattering cross section becomes:

$$
\sigma_{\pi}=\pi r^{2} \Delta \varphi^{2} \beta_{o} \Delta r\left(\frac{P_{S}(r)-P_{B}(r)}{P_{B}(r)-\bar{n}}\right)
$$

where $\mathrm{P}_{\mathrm{S}}$ denotes the power return from both a spike and the background scattering, $\mathrm{P}_{\mathrm{B}}$ denotes the power return the background scattering only and $\bar{n}$ is noise offset derived from average return values beyond $5 \mathrm{~km}$. The background return is calculated from:

$$
P_{B}\left(r_{j}\right)=\frac{1}{4}\left(P\left(r_{j-6}\right)+P\left(r_{j-5}\right)+P\left(r_{j-4}\right)+P\left(r_{j-3}\right)\right),
$$

where $\mathrm{j}$ is the range index for the spike return. The response time of the lidar receiver is limited and large spike returns are followed by a short decay in the signal, which impacts the next range measurement; therefore, to avoid biasing the data, the background signal is derived from data which occurs before the spike. The magnitude of return bins after spike returns is also checked to ensure that the return decreases to values which are close to the pre-spike background. In addition, the resulting spike observations are filtered to remove duplicate measurements of large spike returns at consecutive range bins and from profiles at the subsequent measurement times with the same ranges.

This paper uses Eq. 5 to estimate the backscattering cross section. Most of the variables are determined by measurement and the errors associated with the variables are known and limited. The largest error is associated with determining a value for the volume backscatter coefficient. This value is assumed to be constant and is selected from the report written by Wright et al. [9] which give the coefficient as a function of wavelength and environmental conditions. The error in the value of backscatter coefficient could be as large as an order of magnitude. As a result, the error in value of the derived backscatter cross section could also be as large of as an order of magnitude. Variations of volume backscattering depend strongly on the variations relative humidity [10]. From the estimated humidity variations during the measurement period, the errors caused by variations in volume backscatter coefficient are expected to be less that a factor of two.

\section{MEASUREMENT}

On 17 April 2008, lidar observations, which were by NRL Eye-safe Volume Imaging Lidar system made over the Chesapeake Bay in a clear air boundary layer, detected 1145 spikes in 20 minutes at ranges between 1.5 and $2.25 \mathrm{~km}$. The winds on this day reported by NOAA at Thomas Light (which is twenty kilometers North of lidar) were approximately $4 \mathrm{~m} / \mathrm{s}$ from the 
South and the wind direction was almost perpendicular to the trajectory of the lidar transmit beam. Since the lidar operate at $10 \mathrm{~Hz}$, the wind can move the aerosols from one lidar measurement volume before the next lidar measurement is made. During these measurements the lidar was aimed at fixed elevation and azimuth. Figure 1 shows spikes typical for this day, which are also typical of the "clear-air" spikes detected by the eye-safe lidar over the last few years. While we have observed hundreds of spike returns, the probability of detecting spikes in any individual waveform remains low.

Equation (5) was used to estimate the backscattering cross section. For this calculation, the volume backscatter coefficient is assumed to be $10^{-6} \mathrm{~m}^{-1} \mathrm{sr}^{-1}$ [9]. In Fig. 1, the larger spike represents one of the largest spikes detected during these measurements. In this case, the ratio of the power returns within the bracket is 18.2 and the backscattering cross section is 0.08 $\mathrm{mm}^{2} \mathrm{sr}^{-1}$. The smaller spike in Fig. 1 has a ratio of 0.6 with a backscattering cross section of $0.003 \mathrm{~mm}^{2} \mathrm{sr}^{-1}$.

The total density of spike measurements (with backscattering cross sections magnitudes greater than $0.003 \mathrm{~mm}^{2} \mathrm{sr}^{-1}$ ) was calculated and compared reported densities for radar observations. Each lidar measurement range bin (1.5 m long) is considered to be an independent measurement; thus there are five hundred measurements for each lidar profile. Over the measurement period interval, over ten thousand profiles were recorded. Each measurement volume is approximately $0.04 \mathrm{~m}^{3}$ and, with about five million observations, the total measurement volume is about $2.0 \times 10^{5} \mathrm{~m}^{3}$. (The measurements at individual range bins are assumed to independent of each other which may be true for weaker spikes, but not always true for stronger spikes that can impact more than one range bin.) The target density is about $5.7 \times 10^{-3}$ spikes per cubic meter which is significantly higher than the density ( $5 \mathrm{x}$ $10^{-6} \mathrm{~m}^{-3}$ ) reported by Martin and Shapiro [11] for measurements made by a $9.3 \mathrm{GHz}$ radar. This radar density is the same as the density observed by the lidar for particulates with backscattering cross sections larger than approximately $0.01 \mathrm{~mm}^{2} \mathrm{sr}^{-1}$.

Figure 3 shows the probability distribution of spikes as a function of backscattering cross section. The probability distribution has a tail that extends beyond the spikes caused by Gaussian noise. The Gaussian noise is caused by an analog background signal (primarily sky and electronic noise) and the tail that extends beyond the Gaussian noise is backscatter from particulates. A power law distribution was fit to this tail:

$$
p_{i}=a\left(\Delta P_{i}^{\prime}\right)^{m},
$$

where $p_{i}$ denotes the probability of finding a spike in the $i^{\text {th }}$ measurement bin, a is the amplitude, $\Delta \mathrm{P}_{\mathrm{i}}^{\prime}$ is the size of the $\mathrm{i}^{\text {th }}$ bin, and $\mathrm{m}$ is the magnitude of power law. An uneven, geometric-progress bin size is used to generate the histogram:

$$
\Delta P^{\prime}{ }_{i}=\Delta P^{\prime}{ }_{o} X^{i},
$$

where $\Delta \mathrm{P}_{0}$ is the size of the smallest bin size and $\mathrm{X}$ is the step size of the geometric progression. The bin sizes vary between 0.003 and $0.08 \mathrm{~mm}^{2} \mathrm{sr}^{-1}$. The geometric bin size is used to improve the bin statistics for the largest spike returns. However there are empty bins for many of the large spike return values. We used a least squares algorithm to fit the data. The minimum bin size used in the fit has a spike magnitude of $0.003 \mathrm{~mm}^{2} \mathrm{sr}^{-1}$. The largest bin size is smaller than the first empty bin (i.e. the smallest bin which did not have any recorded spikes); the maximum cross sectional magnitude used is about $0.02 \mathrm{~mm}^{2} \mathrm{sr}^{-1}$. The bins with the largest spikes (which are over $0.02 \mathrm{~mm}^{2} \mathrm{sr}^{-1}$ ) are larger than the smallest empty bin and are not used in the fitting process. 


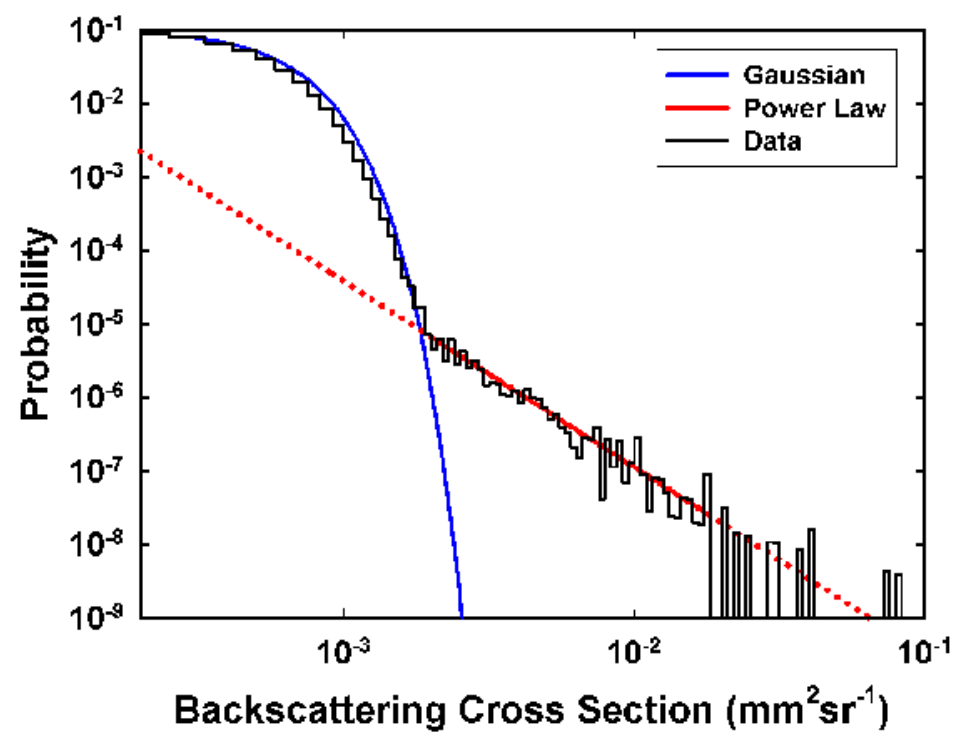

Fig. 3. A histogram of probability distribution of spikes is shown as a function of the backscattering cross section. The blue curve shows a Gaussian distribution fit to cross sections smaller than $0.003 \mathrm{~mm}^{2} \mathrm{sr}^{-1}$ and red curve shows a power law fit to sizes larger than $0.003 \mathrm{~mm}^{2} \mathrm{sr}^{-1}$ and less than $0.02 \mathrm{~mm}^{2} \mathrm{sr}^{-1}$. The dotted line extensions to red curve are provided for visualization purposes only and not derived from any fit beyond the above mentioned data range.

\section{SUMMARY}

This paper reports on lidar measurement of spike returns made over the Chesapeake Bay. Using Eq. (5), the range of backscattering cross sections is between 0.003 and $0.08 \mathrm{~mm}^{2} \mathrm{sr}^{-1}$. While these cross section can not be directly compared with the cross section sizes reported by Martin and Shapiro [11] for radar systems, the lidar-observed measurement density ( $5.7 \mathrm{x}$ $10^{-3}$ spikes per cubic meter) is larger than the density reported by radar systems. The larger measurement density can be explained in part by the sensitivity of the lidar to "targets" with smaller cross sections.

The measurements in this paper are derived solely from remote sensing measurements. We do not have any in situ or physical measurements of the large particulates, which cause the spike returns, nor do we know the shape or composition of the particles. The errors associated estimating the cross sections associated with the spike returns are large (possibly an order of magnitude). However the magnitude of spike returns is consistent with returns from biological particulates (such as insects) or ultra-giant aerosols (UGA) [12].

Published results [13] suggest that high power millimeter wave radars can detect both hard target and diffuse atmospheric features associated with refractive structures. Since lidar does not detect spike returns from diffuse refractive structures, joint lidar-radar measurements of spike returns provide a technique for separating of radar spike returns from "spot angels" or hard target scattering from more diffuse returns from atmospheric refractive structures. Lidar measurements can provide a density measurement for larger aerosols, which are potentially a source of atmospheric clutter for millimeter radars. Birds and insects can move at speeds that differ from the wind speed and, thus, represent a source of systematic error for activemicrowave wind sounders -- lidar-detected spike returns may represent a way to differentiate between moving targets (like birds and insects) and large particulates which follow the air's 
motion. If, indeed, some lidar spike returns are caused by UGA, the measurements are potentially significant, since UGA could significantly impact warm rain initiation [14]. Few studies of UGA have been made and lidar measurements may provide a technique for tracking UGA and determining their atmospheric residency times. For the above reasons, we think that lidar spike returns merit further study.

If future measurements are made, the limitations and inaccuracies of the observations reported in this paper should be reduced. Calibrated targets should be used to ensure that the lidar spike data can be more accurately converted to cross section size. In situ measurements of the aerosol spectra should be made to reduce the errors in estimating extinction and backscatter. Joint lidar and in situ aerosol collection should be made to provide detailed information on the characteristics of the particulates which are causing the spike returns.

\section{Acknowledgments}

The funding for this research was provided by the Office of Naval Research.

\section{References}

[1] H. Ottersten, "Atmospheric structure and radar backscatter to clear air," Radio Sci. 4, 1179-1193 (1969) [doi: 10.1029/RS004i012p01179].

[2] D. Lack and G. C. Varley, "Detection of birds by radar," Nature 156, 443-446 (1945) [doi:10.1038/156446a0].

[3] K. M. Glover, K. R. Hardy, T. G. Konrad, W.N. Sullivan, and A. S. Michaels, "Radar observations of insects in free flight," Sci. 154, 967-972 (1966) [doi:10.1126/science.154.3752.967].

[4] R. J. Munick, "Turbulent backscatter of light," J. Opt. Soc. Am. 55, 893-893 (1965) [doi:10.1364/JOSA.55.000893].

[5] W. P. Hooper and L. U. Martin, "Scanning lidar measurements of surf-zone aerosol generation," Opt. Eng. 38, 250-255 (1999) [doi:10.1117/1.602083].

[6] W. P. Hooper, G. M. Frick, and B. P. Michael, "Using backward Raman scattering from coupled deuterium cells for wavelength scattering," Opt. Eng. 48, 084302 (2009) [doi:10.1117/1.3204230].

[7] R. T. H. Collis, "Lidar," Appl. Opt. 9, 1782-1788 (1970) [doi:10.1364/AO.9.001782].

[8] R. M. Measures, Laser Remote Sensing: Fundamentals and Applications, Krieger, Malabar (1992).

[9] M. L. Wright, E. K. Proctor, L. S. Gasiorek, and E. M. Liston, "A preliminary study of air-pollution measurements by active remote sensing techniques," NASA Langle Research Center, NASA CR-132724 (1975) (available from http://ntrs.nasa.gov).

[10] J. W. Fitzgerald, "Effect of relative humidity on the aerosol backscattering coefficient at 0.694- and 10.6- $\mu \mathrm{m}$ wavelengths," Appl. Opt. 23, 411-418 (1983) [doi:10.1364/AO.23.000411].

[11] W. J. Martin and A. Shapiro, "Discrimination of birds and insects radar echoes in clear air using high-resolution radars," J. Atmos. Oceano. Techn. 24, 1215-1230 (2007) [doi:10.1175/JTECH2038.1].

[12] S. Lasher-Trapp and J. P. Stachnik, "Giant and ultragiant aerosol particle variability over the eastern great lakes region," J. Appl. Meteorol. Cli. 46, 651-659 (2007) [doi:10.1175/JAM2490.1].

[13] M. T. Ngo and G. Linde, "Clear-air backscatter from the lower atmosphere using a high-power, millimeter wave radar," 33rd Int. Conf. on Infrared, Millimeter, and Terahertz Waves, 454-455 (2008) [doi:10.1109/ICIMW.2008.4665646].

[14] D. B. Johnson "The role of giant and ultra-giant aerosols in warm rain initiation," $J$. 
Atmos. Sci. 39, 448-460 (1982) [doi: 10.1175/1520-0469(1982)039>0448:

TROGAU $<2.0 . C O ; 2]$.

William P. Hooper received his BA in physics from Colorado College in 1970. After six years in the U.S. Army, he attended the University of Wisconsin, where he received his MS in Meteorology in 1982 and did his thesis on lidar wind measurements. Since 1982, he has been at the Naval Research Laboratory, and he currently is the head of the Aerosol and Lidar Physics Section. His research involves lidar, primarily as a tool for characterizing the atmosphere immediately above the ocean.

Glendon M. Frick graduated from the University of Missouri, Rolla with a MS degree in mechanical engineering in 1981. He has since been employed at the US Naval Research Laboratory, where he worked on numerous atmospheric aerosol research and remote sensing projects. 